\title{
Question of Parity Non-Conservation in Weak Interactions
}

\author{
Zuodong Sun ${ }^{*}$ \\ Ya'ou Brain Science Institute of Heilongjiang province, Harbin 150090, China
}

\begin{abstract}
:
In order to reasonably explain the phenomenon of cell bioelectricity, we proposed the conservation law of cell membrane area, established the ion inequality equation, and therefore paid attention to the mystery of " $\theta-\tau$ ". We researched and analyzed the " $\theta-\tau$ " mystery, discussed the parity non-conservation in weak interactions, suggested possible experiments to test the parity non-conservation in weak interactions, and gave our research and analysis conclusions: The experimental scheme proposed by C. N. Yang and T. D. Lee in the hypothesis cannot be used as a positive evidence of whether the weak interaction parity is conserved, nor can it directly answer whether $\theta$ and $\tau$ in the " $\theta-\tau$ " mystery are the same particle; The $\operatorname{Co}^{60} \beta$ decay experiment such as C. S. Wu is a pseudo-mirror experiment, and it has not overturned the so-called "parity conservation law" or proved the "parity non-conservation" in weak interactions; The " $\theta-\tau$ " mystery is a "man-made" mystery. $\theta$ and $\tau$ are two different particles, which may be the result of the same precursor particle being divided into two. Parity conservation or non-conservation under mirror image has no physical significance. The work of C. N. Yang, T. D. Lee, C. S. Wu et al. have brought quantum physicists from the "Little black house" to the "Big black house" or "smaller black house". The right and wise choice is to go back through "the door that came in". With the development of science today, it is time for some contents to reform from the bottom.
\end{abstract}

Keywords: mystery of $\theta-\tau$; question of parity non-conservation; C. N. Yang; T. D. Lee; "origami windmill" model

In 1954, a problem called the $\theta$ - $\tau$ problem appeared. Scientists found that the mass and lifespan of the two mesons $\theta$ and $\tau$ are almost the same, and most people think that they are the same particle. However, when $\theta$ decays, $2 \pi$ mesons are produced, and when $\tau$ decays, 3 $\pi$ mesons are produced, which shows that $\theta$ and $\tau$ are not the same particle. If $\theta$ and $\tau$ are the same particle, it violates the law of conservation of parity; if they are not the same particle, their mass and life span are almost the same. This is the mystery of " $\theta-\tau "[1,2]$.

In 1956, C. N. Yang and T. D. Lee published a paper[1,2] in which they proposed the hypothesis of "parity is not conserved in weak interactions" and suggested possible experiments to test whether parity is conserved in weak interactions; C. S. Wu et al. first made a judgment Sexual experiment[3-5], namely: use two sets of "mirror images" of each other to observe the $\beta$ decay of $\mathrm{Co}^{60}$. According to C. S. Wu's experimental results, people believe that the mystery of " $\theta-\tau$ " has been solved.

In 2020, we proposed the conservation law of cell membrane area and established the ion

* Corresponding author: Zuodong Sun (E-mail: sunzuodong@pai314.com). 
inequality equation, which reasonably explained the phenomenon of cell bioelectricity[6-12]. Although cell bioelectricity is not in the scope of the physics definition of weak interaction force, we still pay attention to the mystery of " $\theta-\tau$ ". We traced the roots of this, sorted out the work of theoretical and experimental physicists closely related to the " $\theta-\tau$ " mystery, especially the work of C. N. Yang, T. D. Lee, C. S. Wu, etc., and the research analyzed the mystery of " $\theta-\tau$ ", discussed the parity non-conservation in weak interactions, and suggested possible experiments to test the parity non-conservation in weak interactions.

\section{Are $\theta$ and $\tau$ the same kind of particles?}

C. N. Yang et al. analyzed[1] that in strong and electromagnetic interactions, existing experiments show the conservation of parity with high accuracy; however, the parity conservation of weak interactions is still an extrapolation hypothesis, and there is no experimental evidence to support it. ...Current experimental limit of parity non-conservation $\mathcal{F}^{2}<3 \times 10^{-13}$, this is the most accurate verification of parity conservation in strong and electromagnetic interactions. However, even this high accuracy is not enough to provide experimental proof of parity conservation in weak interactions. For this suggestion, an accuracy of $\mathcal{F}^{2}<10^{-24}$ is required.

C. N. Yanget al. believe[18] [19] ${ }^{55}$ that the higher the accuracy, the more proof $\theta$ and $\tau$ Is the same particle. Within the experimental error range (about a few percent), the two particles have exactly the same lifetime and similar mass. At present, there are more than 1000 experimental sites, and the general view is $\theta$ and $\tau$ Not the same particle. However, this cannot be hastily concluded, because its mass accuracy is from 2 to 10 electrons, or a fraction of one percent, and its lifetime accuracy is $20 \%[20][21]^{159-160}$.

We believe that another very important indicator that may determine that $\theta$ and $\tau$ are not the same particle has not been taken seriously, namely: the size and density of the nucleus. It has been known in the alphaparticle scattering experiment that $[22]^{220-224}$ the nucleus is at the center of the atom, and its linearity is only one ten thousandth of that of the atom, but its mass accounts for more than $99.9 \%$ of the atom. The experimentally determined nucleus radius can be expressed by the following formula:

$$
R=r_{0} A^{1 / 3}
$$

Among them, $r_{0}$ is the coefficient determined by experiment, and $A$ is the mass number of the nucleus. The $r_{o}$ value obtained by the electromagnetic method is about $1.2 \times 10^{-15} \mathrm{~m}$, and the rovalue obtained by the nuclear force method is about $1.4 \times 10^{-15} \mathrm{~m}$. From the radius of the nucleus, the approximate volume of the nucleus is:

$$
V=\frac{4}{3} \pi R^{3}=\frac{4}{3} \pi r_{0}^{3} A
$$


The mass of the nucleus is approximately the mass of the atom $M=A / N_{\mathrm{A}}$, so the density of the nucleus:

$$
\rho=\frac{M}{V}=\frac{3}{4 \pi r_{0}^{3} N_{\mathrm{A}}}=2.3 \times 10^{17} \mathrm{~kg} \cdot \mathrm{m}^{-3}
$$

Where NA is Avogadro's constant. It can be seen that the density of various nuclei is close to a constant and very large, about $10^{13}$ times the density of iron $7.9 \times 10^{3} \mathrm{~kg} \cdot \mathrm{m}^{-3}$. Because of this, the "slightly" difference in the size and density of the nucleus is sufficient to prove that $\theta$ and $\tau$ are not the same particle. The experimental accuracy is not up to the point, or the mass and life are "almost" the same, which is not a sufficient reason to conclude that $\theta$ and $\tau$ are the same particle. Based on the conservation of angular momentum and parity, Dalitz has conducted statistics and analysis on the decay products of $\tau[23-26]$, and strongly believes that $\theta$ and $\tau$ are at least two different charged particles.

The conclusions of our analysis support the results calculated by Dalitz. As long as the masses and lifetimes of $\theta$ and $\tau$ are different, it is sufficient to be a clear evidence that $\theta$ and $\tau$ are not the same particle, not to mention produces two $\pi$ mesons when $\theta$ decays, and produces three $\pi$ mesons when $\tau$ decays. The quality and life measured by the experiment are "almost the same", not "exactly the same." The experimental results of "measures becoming more and more accurate" should be more accurate to prove the difference in quality and life of $\theta$ and $\tau$, and that $\theta$ and $\tau$ are not the same particle, instead of what C. N. Yang et al. think, "the higher the accuracy, the more it can be proved $\theta$ and $\tau$ Is the same particle". We believe that $\theta$ and $\tau$ result from the "split into two" of the same precursor particle, that is, $\theta$ and $\tau$ always appear at the same time and accompany each other. There are as many $\tau$ as there are $\theta[2,18]$, and the probability of occurrence is $50 \%$ each.

\section{Isn't the parity in weak interactions conserved?}

C. N. Yanget al. hypothesized that $[1,19]$ "parity is not strictly conserved, and $\theta$ and $\tau$ are two different decay modes of the same particle, and their mass and life span must be the same. "They suggest experiments: use $\mathrm{Co}^{60}$ for $\beta$ decay, and measure the angular distribution of the emitted electrons in the $\beta$ decay of $\mathrm{Co}^{60}$ polarized nuclei. Let $\theta$ be the angle between the orientation of the mother nucleus and the electron momentum, the asymmetry of the distribution of $\theta$ and $180^{\circ}-\theta$ constitutes a positive evidence of parity non-conservation in $\beta$ decay.

C. N. Yang interpreted the plan they gave[19] $]^{56-57}$ : As a possible method to solve the $\theta-\tau$ problem, it is recommended that experiments should be used to test whether the principle of left-right symmetry will be violated in weak interactions. The principle of the test is extremely simple, only two sets of experimental devices that are mirror images of each other need to be established. They must include weak interactions, and they must be different from 
each other. If these two devices are identical to each other, they will always produce the same results, and experiments cannot be used to test left-right symmetry. Then check whether the two devices always produce the same results. If they do not always produce the same results, we have obtained a definite proof against the principle of left-right symmetry in this experiment.

C. S. Wu, E. Ambler, R. W. Hayward, D. D. Hoppes and R. P. Hudson conducted the first such experiment in 1956[3](Fig. 1 and Fig. 2). The Co nucleus decays due to weak interactions, and the decay products are then counted.It must be noted here that the current flowing in the coil is a necessary factor in the experiment. Without these currents, the two devices on both sides of the mirror will be the same and will always produce the same result. The result of the experiment is that there is a very large difference between the readings on the two meters shown in Figure 2. Since the properties of the other parts of the instrument are symmetrical, the asymmetry can only be caused by the decay process of $\mathrm{Co}^{60}$, which is caused by weak interaction.
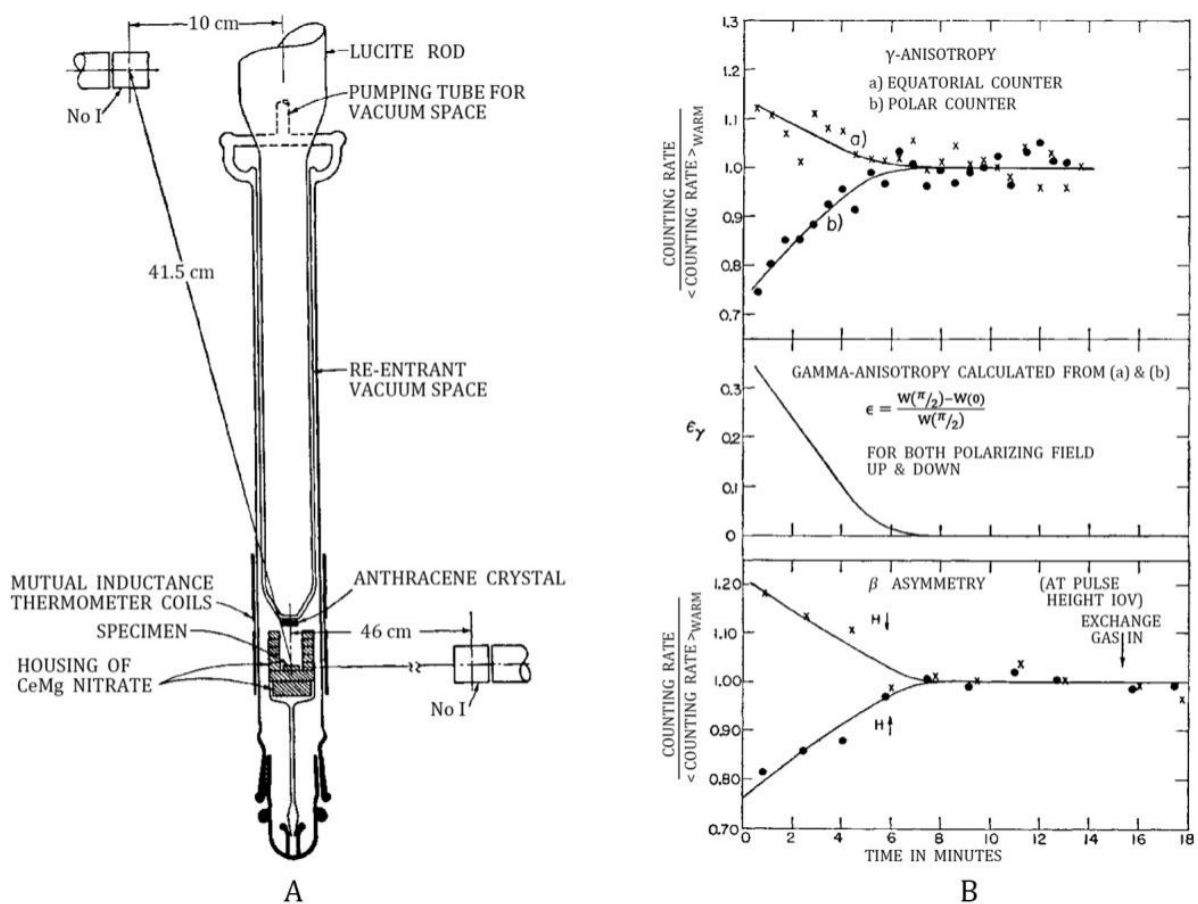

Fig.1 Mirroring experimental device and experimental results[3]

A. $\mathrm{Co}^{60} \beta$ decay experimental device;B. $\mathrm{Co}^{60}$ beta decay experiment results.

We believe that the experimental scheme given by C. N. Yang et al. Can not test whether parity is conserved in weak interaction. We therefore suggest that, on the basis of $\mathrm{C}$. S. Wu 's experiment, the following experiments should be further conducted: (1)Using the same set of experimental equipment, the direction of the current in the coil remains unchanged, and the $\beta$ decay of $\mathrm{Co}^{60}$ is observed twice, and the experimental results are predicted to "will not always produce the same results"; (2)Using the same experimental device, the current 
direction in the coil is different, and the $\beta$ decay of $\mathrm{Co}^{60}$ is observed twice, the predict experimental results and "Wu's current experimental results should be similar, there will be no big differences"; (3)The direction of the current in the two sets of experimental devices and coils remains unchanged, and the $\beta$ decay of $\mathrm{Co}^{60}$ is observed twice to predict that the experimental results "will not always produce the same results";(4)Distribute $\mathrm{Co}^{60}$ uniformly in the cerium magnesium nitrate crystal to observe the anisotropy of $\gamma$-rays. The result should be very different from the experimental observation results of C. S. Wu et al., or obtain diametrically opposite results within the allowable range of experimental error.

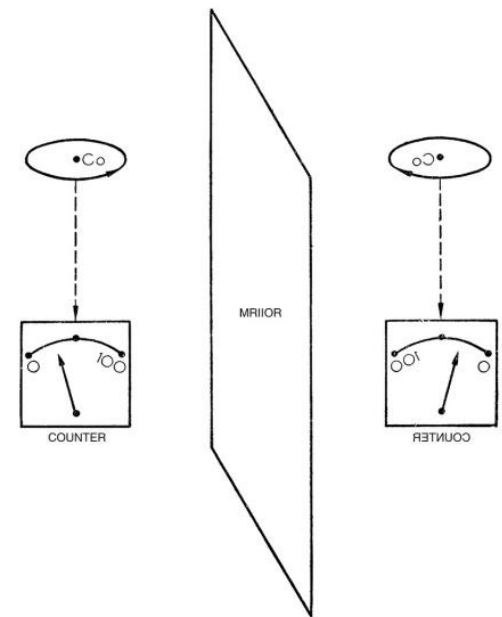

A

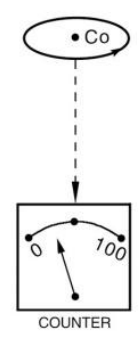

Fig.2 Principle of mirroring device[19] $]^{56-60}$

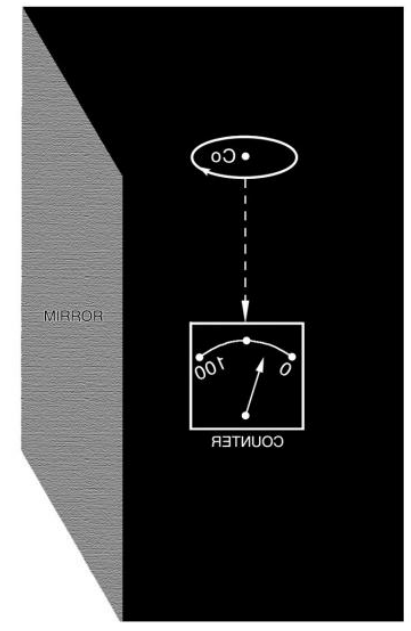

B

A. Principle of $\mathrm{Co}^{60} \beta$ decay experimental device; B. The new definition of mirror reflection.

The four experiments suggested above can be understood as falsification experiments of non-conservation of parity in weak interactions. As long as one of the experimental results meets our prediction, it is sufficient to prove that C. S. Wu's experimental results are not sufficient to support the hypothesis proposed by C. N. Yang et al.."Since the properties of the other parts of the instrument are symmetrical, the asymmetry can only be caused by the decay process of $\mathrm{Co}^{60}$, which is caused by weak interaction." C. N. Yang's judgment is also invalid.

In C. N. Yang's paper, regarding the most general form of Hamiltonian[1], Dr. R. B. Curtis and Dr. M. Morita pointed out the error in the formula (A.4). Among them, Dr. M. Morita was a member of C. S. Wu's research group in October 1956[4]. The most general form of Hamiltonian in the appendix of the paper (A.4):

$$
\begin{aligned}
\alpha \xi=\frac{1}{3}\left(\left|C_{T}\right|^{2}-\right. & \left.\left|C_{A}\right|^{2}+\left|C^{\prime}{ }_{T}\right|^{2}-\left|C^{\prime}{ }_{A}\right|^{2}\right)\left|M_{G . T .}\right|^{2} \\
& -\left(\left|C_{S}\right|^{2}-\left|C_{V}\right|^{2}+\left|C^{\prime}{ }_{S}\right|^{2}-\left|C_{V}^{\prime}\right|^{2}\right)\left|M_{F .}\right|^{2}
\end{aligned}
$$


Corrected to

$$
\begin{aligned}
\alpha \xi=-\left(\left|C_{S}\right|^{2}-\right. & \left.\left|C_{V}\right|^{2}+\left|C^{\prime}{ }_{S}\right|^{2}-\left|C_{V}^{\prime}\right|^{2}\right)\left|M_{F .}\right|^{2} \\
& +\frac{1}{3}\left(\left|C_{T}\right|^{2}-\left|C_{A}\right|^{2}+\left|C^{\prime}{ }_{T}\right|^{2}-\left|C_{A}^{\prime}\right|^{2}\right)\left|M_{G . T .}\right|^{2} \\
& +2 \operatorname{Re}\left\{i \frac { Z e ^ { 2 } } { \hbar c p } \left[\left(C_{S} C_{V}^{*}+C^{\prime}{ }_{S} C^{\prime}{ }_{V}^{*}\right)\left|M_{F .}\right|^{2}\right.\right. \\
& \left.\left.-\frac{1}{3}\left(C_{T} C_{A}^{*}+C^{\prime}{ }_{T} C^{\prime}{ }_{A}^{*}\right)\left|M_{G . T .}\right|^{2}\right]\right\} .
\end{aligned}
$$

C. N. Yang believes that this change does not affect the narrative in the article, nor does it affect the rest of the appendix. Since it has no effect on the paper, why should such a complicated mathematical equation be written into the paper?

In fact, the idea of non-conservation of parity in weak interactions was not first proposed by C. N. Yang et al.. The first paper on this issue was written by Purell and Ramsey[27]. It dealt with the possibility of the electric dipole moment of elementary particles, but it was only a kind of Possibilities are raised rather than solutions to a real problem. They pointed out that the main theoretical objection to the existence of that electric dipole moment is based on parity conservation, but for elementary particles and nuclei the parity conservation has not been tested[1,27-29]. Interestingly, this paper was incorrectly cited by Yang et al. They pointed out:"The upper limit of the most accurate electric dipole moment so far is $e \times\left(5 \times 10^{-20} \mathrm{~cm}\right) \ldots$ This is also the most accurate verification of the conservation of parity in strong and electromagnetic interactions."Their note 6 points out Purell and Ramsey's papers cited in Ramsey's molecular beam[2] $]^{323}[27,28]$. Although the original paper did not give any quantitative results of the neutron electric dipole moment, but only pointed out that the experiment has made progress, the book says that this result is contained there [30]. Therefore, it is clear that Yang has never read this original text, and T. D. Lee confirmed this[21 $]^{221}$.

In 1952 Wick et al.'s paper, there are further opinions on parity non-conservation[29]: Even if our thinking does not fundamentally change due to this, we believe that it is useful to pay certain attention to this matter, for example, it can prevent people from taking "theorems" as "hypotheses" or "theorems" as some kind of The "impossible" theoretical form is abandoned. Previously, the former Soviet physicist Landau also put forward the compound inversion theory[31].

\section{Are C. S. Wu's experiments "mirror images" of each other?}

Regarding how C. S. Wu's two sets of experimental devices mirror each other is not mentioned in the experimental report of $\mathrm{Wu}$ et al[3], nor in the experimental scheme proposed by C. N. Yang and T. D. Lee[1]. It is known from related literature that $[19,36]$ by changing the direction of the input current of the magnetic induction coil, corresponding to 
the left-right transformation of the mirror image, the right-handed screw rule is applied, and the right-handed screw rule is converted into the left-handed screw rule[15] ${ }^{270}$. How to ensure the spin direction of the $\mathrm{Co}^{60}$ nucleus? How to ensure the same symmetry up and down?This is how $\mathrm{Wu}$ interpreted it[4], "Two $\mathrm{Co}^{60}$ radiation sources have been prepared: one is to grow a layer containing $\mathrm{Co}^{60}$ on one side of a perfect single crystal of cerium magnesium nitrate. This crystal layer is about 0.002 inches thick and contains a few microbits of radioactivity; The other is to evenly distribute $\mathrm{Co}^{60}$ in the cerium magnesium nitrate crystals to study the anisotropy of $\gamma$ rays."

However, in the experiment of decay parity conservation, the first option was chosen: The sample uses a good single crystal of cerium magnesium nitrate, and only a crystal layer containing $\mathrm{Co}^{60}$ grows on its upper surface. The polarization of the $\mathrm{Co}^{60} \beta$ source obtained by the demagnetization method can be as high as $65 \%$.Since the magnetic properties of electrons are easily saturated at low temperatures, a magnetic field of several hundred Gauss is sufficient. The nuclear orientation will automatically be formed immediately. This is because the low-energy beta particles move in a spiral shape tightly along the direction parallel or anti-parallel to the magnetic field lines[3,4]. It is observed that the asymmetry of $\mathrm{Co}^{60} \beta$ decay does not change with the reversal of the demagnetizing field, which means that the emission of particles tends to be in the opposite direction to the direction of nuclear spin.

Our analysis believes that the principle of mirroring only refers to left-right symmetry. If the mirroring experiment is implemented, in addition to left-right symmetry, it is necessary to ensure that the up and down, front and back directions cannot be changed. The $\operatorname{Co}^{60} \beta$ source has a polarization degree of $65 \%$, so how does the other $35 \%$ of the $\operatorname{Co}^{60} \beta$ source that have not been polarized decay? How to ensure and determine whether the direction of the $\mathrm{Co}^{60}$ nucleus spin is left rotation or right rotation? Can it be determined by changing the direction of the input current of the magnetic induction coil? Is the spin direction of the $\mathrm{Co}^{60}$ nucleus suitable for applying the right-handed spiral rule, or is it converted from the right-handed spiral rule to the left-handed spiral rule? (Fig.3) If the above question can be answered in the affirmative, then how to explain the " $\mathrm{Co}^{60} \beta$ decay asymmetry that will not change with the reversal of the demagnetizing field" observed by C. S. Wu's experiment? Just like J. Buridan's donkey, C. N. Yang's interpretation of "Mach's confusion" is also very contradictory and far-fetched [32] ${ }^{18-19}$ (Fig. 4).

Therefore, although the radioactive sources in C. S. Wu's two sets of experimental devices are both $\mathrm{Co}^{60}$, the experimental conditions did not truly mirror each other. The difference in the final measurement results is a normal high probability event, and there is no difference or the result of small differences is an abnormal and small probability event. Although C. N. Yang and T. D. Lee repeatedly stated that C. S. Wu's experimental principle was "simple", according to $\mathrm{Wu}$, the preparation process was quite "complex"[4]. 


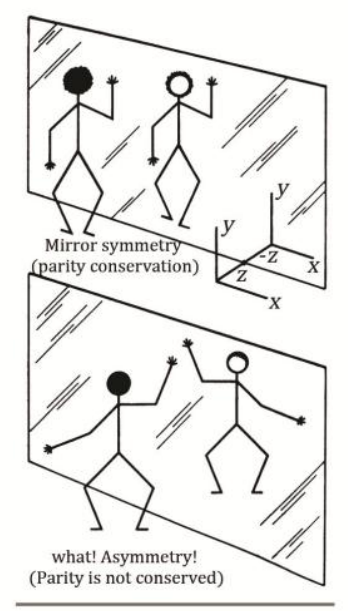

A
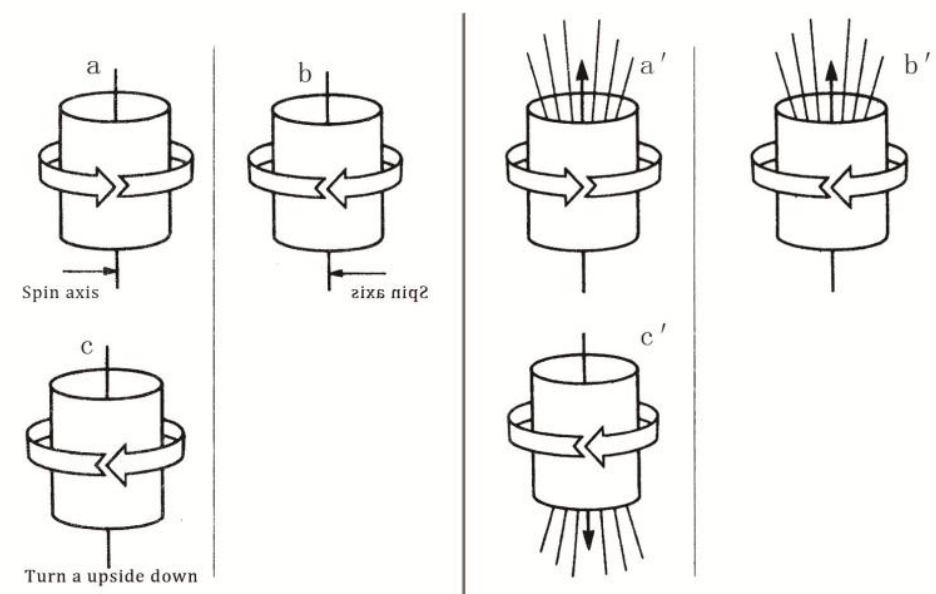

Turn a upside down

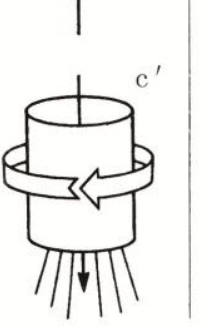

B

Fig. 3 Mirror symmetry and right-handed spiral rule[15] $]^{266-271}$

A. Parity conservation and parity non conservation.

B. Laboratory and Mirror Laboratory. a represents a spinning particle, $\mathrm{b}$ is the mirror image of $\mathrm{a}$, and $\mathrm{c}$ is the upside down of $a$. It is exactly the same as $b$. Therefore, $b$ corresponds to a particle that can be found in nature, and mirror symmetry is observed. a'depicts a decayed $\mu$, the arrow on the spin axis indicates the direction of the radiated electrons, and the radial lines indicate that the electrons are more "right-handed"; b'is the mirror image of a', which is a left-handed decay $\mu$, if experiments show that all $\mu$ decay right-handedly, then b'does not exist in nature. For example, turning a'upside down and c'cannot copy b', and the mirror symmetry is destroyed.

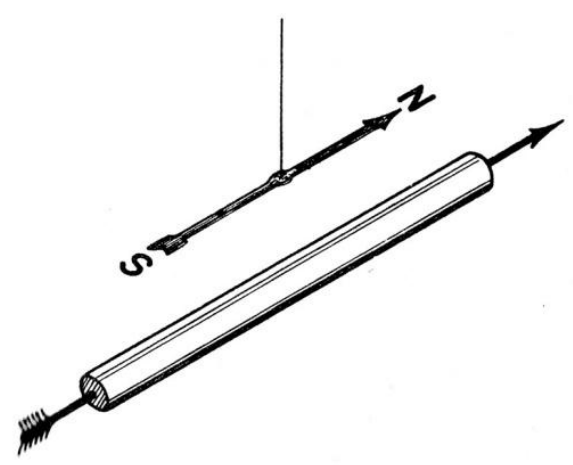

A

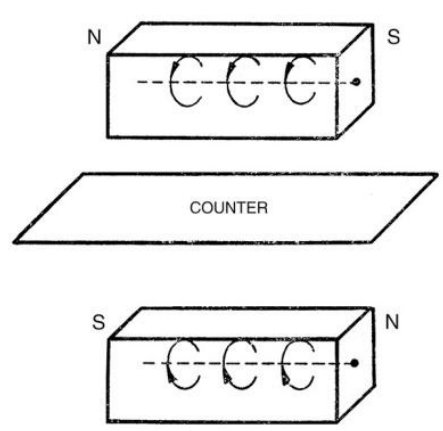

B

Fig. 4 Mach's confusion and C. N. Yang's answers[19] $]^{61-62}$

A. Mach's confusion If the magnetic needle is hung parallel to a wire that is energized in a certain direction, it will turn left or right when it is deflected in a certain direction. Mach said that the magnetic needle must be like Buridan's donkey, refusing to make a decision between left and right;

B. C. N. Yang answers questions Reflection symmetry, based on a deeper understanding of magnetism, shows that this symmetry is only superficial. In the case of reflection, the polarity of the magnet changes, so this reflection symmetry is not true.

There is another very important detail that C. N. Yang deliberately avoided. C. S. Wu once said, "At that time, I was still reluctant to let them (referring to C. N. Yang and T. D. Lee) quote our experimental results, but I promised to give them a positive result soon"[4].

In the experimental report paper published by C. S. Wu et al.[3], the opening emphasized: "In a recent paper on the parity problem of weak interactions, Lee and Yang critically examined experimental information about this problem, ...they put forward some 
experiments on beta decay, hyperon decay, and meson decay, which will provide necessary evidence for whether parity is conserved. In $\beta$ decay, the angular distribution of electrons from the $\beta$ decay of polarized nuclei can be measured. If the asymmetry of the $\theta$ and $180^{\circ}-\theta$ distributions provides clear evidence of non-conservation in $\beta$ decay, this asymmetry effect is already in $\mathrm{Co}^{60}$ Observed under the circumstances". At the end of the paper[3], $\mathrm{Wu}$ emphasized again: "According to Lee and Yang's point of view, the experimental results not only violate the conservation of parity, but also violate the invariance under the charge conjugate. In addition, the invariance under time reversal ..."

In fact, C. S. Wu has never directly stated that her experimental results can be used as positive evidence of whether parity is conserved. However, afterwards, Yang and Lee repeatedly emphasized in their speeches and writings on different occasions that it was Wu's experiment that overturned the "parity conservation"[2,18,34-36][33] $]^{143-159}$. They once commented on C. S. Wu, "As the first author, Chien-shiung denied the law of conservation of parity by experiment for the first time, and also denied the hypothesis of particle-antiparticle symmetry. Symmetry and conservation are the basis of physics, but these two very important laws and assumptions have been overturned by Wu's experiments."'In physics, parity conservation means that left and right are symmetrical. If there are two systems, they are mirror images of each other at the beginning, which means that their initial states are exactly the same, but left and right are different. "[36] In fact, the degree of non-conservation in Wu's experimental data is only $1 \%$ to $2 \%[18]^{208}$.

In the process of weak interaction, whether parity is conserved or not cannot be determined a priori theoretically. Yang and Lee only theoretically analyzed the existence of this possibility. The final verdict is the scientific experiment. At that time, the "up-and-down asymmetry" experiment in the beta decay phenomenon of polarized $\mathrm{Co}^{60}$ nuclei at low temperature done by the American physicist Dr. C. S. Wu was the first of many decisive experiments[2] $]^{7-8}$.

The conclusion of our research and analysis: Wu's $\mathrm{Co}^{60} \beta$ decay experiment is relatively rough, this "simple and complicated" experiment is a pseudo-mirror experiment. The "up and down asymmetry" experiment in the decay phenomenon of the Co ${ }^{60}$ nucleus, the result of asymmetry is not equal to the absence of symmetry. Physics is an experimental science. First of all, the design of the experiment must be scientific and rigorous. Judgmental experiments should be used with caution, don't forget Rutherford's famous advice-don't forget to think.

\section{How is "parity" "non-conserved"?}

To answer this question, we must go back to the source and clarify the relationship between the following basic concepts: invariance and symmetry, parity and conservation, symmetry and left and right symmetry, left and right symmetry and mirror left and right 
symmetry, mirror left and right symmetry and mirror symmetry, mirror symmetry and top and bottom symmetry. Invariance corresponds to conservation, which is the core idea of Noether'stheorem[18 $]^{156}[32]^{39}[37-39]$. Because microscopic particles have even and odd numbers in the energy level transition, this odd and even characteristic of energy levels is called the parity of energy levels[2] $]^{99}$. The motion state of a microscopic particle is described by a wave function. When the space coordinate is inverted, if the wave function remains unchanged, the particle motion state has an even parity, which is represented by the parity quantum number 1 . If the wave function changes, the particle's motion state has an odd parity, which is represented by the parity quantum number -1 . During the transition of microscopic particles, the parity of the initial state is always equal to the parity of the final state[2] $]^{98}$, which is the so-called "parity conservation". Mirror symmetry is also called left-right symmetry, which is discrete rather than continuous symmetry.

The above basic concepts have been repeatedly confused, which is the key to the "non-conservation of parity in weak interaction". The invariance in Noether's theorem hides symmetry, but it is not equivalent to symmetry. An invariance corresponds to a conserved quantity or conservation law. When "invariance" is equivalent to "symmetry", Noether's theorem has been obscured or tampered with. Then, symmetry is equivalent to left and right symmetry, left and right symmetry is equivalent to mirror left and right symmetry, and mirror left and right symmetry is equivalent to mirror symmetry. The result of the exchange of these basic concepts: Mirror symmetry is equivalent to conservation of parity, and mirror asymmetry up and down is equivalent to mirror asymmetry. Therefore, mirror image asymmetry is equivalent to parity non-conservation. During the process of turning the principle of mirror symmetry into the so-called "common sense", the basic concepts were exchanged many times. This is a typical disguised replacement of concept, circular argument, disguised replacement of concept, also known as the Scarecrow fallacy; Circular argument is to wrap the argument in the evidence. For example, how did A become B? A - A(B)-B(A)- B. Turning "invariance" into "symmetry" and "symmetry" into "mirror symmetry" is much more complicated than A to B.

Regarding some of the above basic concepts, C. N. Yang gave a rather contradictory explanation in his biography, "The so-called conservation of parity, simply put, is that the laws of physics are at the deepest level, regardless of left and right. There is no difference on the left and right. In other words, according to this law, the operation of an object and its left and right mirror images are the same. In fact, the law of conservation of parity in real physical action is not limited to 'mirror symmetry'. In the real physical action, it should be the displacement symmetry of the entire space left and right, up and down, and front and back. In quantum mechanics, what is discussed is the parity number conservation of space coordinate variables. "[33]

We believe that no matter whether it is discrete symmetry or continuous symmetry, there is no corresponding conserved quantity in the mirror image. Therefore, there is no "parity 
conservation" in the left-right symmetry of the mirror image, and there are no experimental results that overthrow the parity conservation and the non-conservation of parity. Conservation of parity is a scarecrow, and it still belongs to the category of invariance in Noether's theorem. The so-called parity conservation or non-conservation under the mirror image condition does not produce any physical meaning. After 1957, C. N. Yang repeatedly and deliberately confused these basic concepts in speeches and works on different occasions, making people farther and farther away from the truth[2,5,18,19,33-35]. How to "justify oneself" is probably a task that C. N. Yang will never accomplish in this life.

"Symmetry" is the same as "invariance" in Noether's theorem, and belongs to the "phenomenon of things". They jointly correspond to the "essence of things"--the Conserved quantity or the law of conservation. We should not confuse "symmetry" with "conserved quantity", as there is a fundamental difference between phenomenon and essence. C. N. Yanget al. defines "parity" as "conserved quantity corresponding to left-right symmetry", and "parity of the initial state is always equal to the parity of the final state during the transition of microscopic particles" is defined as "parity conservation", Is to deliberately confuse the concepts of parity and conservation[18,19,36].

We now restore "parity" to "odd-even" and "parity conservation" to "parity invariance", then the definition of parity invariance should be: During the transition of microscopic particles, the parity of the initial state is always the same as the parity of the final state. The conserved quantity corresponding to parity invariance should be the law of conservation of charge.

C. N. Yang has a soft spot for "symmetry". The first paper he wrote when he was a graduate student in China was said to be about the study of symmetry. Extracting "symmetry" from "invariance" and further proposing "symmetry governing interaction", these works originated from Maxwell, Minkowski and Einstein, and were applied and popularized by them. Yang only further "advanced" this kind of symmetry in mathematics[40, 41] (Fig.5).

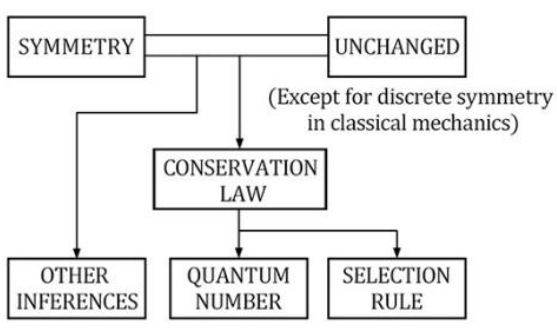

A

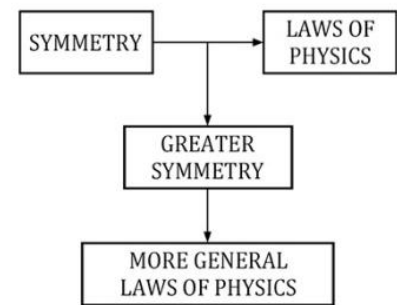

B

\begin{tabular}{|c|c|}
\hline Before Einstein and Minkowski & After Einstein and Minkowski \\
\hline Experiment $\rightarrow$ Field equation $\rightarrow$ Symmetry (Unchanged) & Symmetry $\rightarrow$ Field equation \\
\hline
\end{tabular}

C

Fig.5 Symmetry, invariance and conserved quantities, physical laws[40]

A. Symmetry, invariance and conservation laws; B. Symmetry and physical laws; C. Experiments, field equations and symmetry. 
The Yang-Mills gauge field theory known as the "Holy Grail" of modern physics[18] ${ }^{168-169}$, also known as the non-Abelian gauge field theory, has the same problem.The Yang-Mills gauge field theory was born out of Maxwell's equations, replacing the $U(1)$ group of Maxwell's equations with the SU(2) group in group theory, and the U(1) group is the Abelian group. The originals belong to O. Klein, as well as Hermann Weyl, Vladimir Fork and Fritz London. T. D. Lee's evaluation of the Yang-Mills gauge field theory is a "rediscovered" after Pauli[2] ${ }^{160-161}$. On July 21-25, 1953, Pauli's manuscript "Meson-Nucleon Interaction and Differential Geometry" was formed. It was not officially published "because the field particle mass" was zero; in 1938, O. Klein proposed this Equation, some people say that C. N. Yang copied O. Klein's results[2].

\section{How is parity non-conservation "proven"?}

In fact, C. N. Yang himself admitted that there is nothing to say about the non-conservation of parity in weak interactions, just a guess[33].The paper does not claim that parity is indeed unconserved in weak interactions[33 $]^{142-155}$, and C. S. Wu has never directly stated that her own experimental results can be used as positive evidence of whether parity is conserved. Then, the problem arises, why is the parity in weak interactions not conserved?

On April 3-7, 1956, at the seminar on the theoretical interpretation of new particles, Feynman replaced Block and put forward the hypothesis to solve the " $\theta-\tau$ " mystery; On April 8th or 9th, T. D. Lee and Jack Steinberger $[2,14]$ discussed Block's hypothesis. Lee had a sudden inspiration. To solve the " $\theta-\tau$ " mystery, he must first leave the $\theta-\tau$ system and measure pseudoscalar quantities other than $\theta-\tau$; In early May, Steinberger reported T. D. Lee's hypothesis at the academic report meeting, and C. N. Yang expressed his opposition on the spot; At the end of April or the beginning of May, Yang and Lee discussed the " $\theta-\tau$ " mystery in a Tianjin (or Shanghai) restaurant $[2,18]$. At a key point, Yang "epiphany" the generation and decay reaction chain of $\Lambda^{0}$; In May, C. S. Wu began to prepare for the experiment, and Lee wrote the manuscript "Questioning the Conservation of Parity in the Weak Interaction of Strange Particles"; At the end of May, Lee handed the manuscript of the paper to Irene Tramm for typing, and then sent it to Yang; Yang and his wife should retype, handwriting mathematical equations, change the title of the paper and submit the paper to "Physics Review"; On June 22, "Physics Review" received the paper; on October 1, the paper was published in "Physics Review" 104 issue.

On the morning of January 4, 1957, C. S. Wu informed Lee and Yang that he had observed preliminary experimental results, and urged not to talk to the outside world because the experimental process was to be verified; At noon on January 4th, Lee celebrated his work ahead of time and revealed to the physicists (10-15 people) who were dining together on the Chinese Food Lunch Day at Columbia University, saying that Wu was observing a huge effect; 
On January 5, Yang sent a telegram to Oppenheimer who was vacationing in the Caribbean Sea, reporting Wu's preliminary experimental results; On January 9, Wu "confirmed" the experimental results; On January 10, Wu officially notified Lee and Yang of the experimental results; On January 11, Wu reported the experimental results in the Department of Physics of Columbia University; On January 15th, Columbia University held an exception for a press conference. Rabi rushed back from the field to preside over the meeting and announced the news; On January 16, the New York Times published news on the front page, and a long article reported the incident in detail. The title is "Declaring that a basic concept in physics was overturned by experiments"; On January 17, the editorial department's "Surface and Facts" was published on the front page, which shocked the physics community; On February 2, the American Physical Society held a special meeting at the New York Hotel to report the "discovery" of parity non conservation, and the number of participants was as high as 3110; In early October, the Nobel Prize in physics was awarded to T. D. Lee and C. N. Yang, which is considered to be the "fastest" prize in the history of the Nobel Prize.

On the other hand, the relevant partners and cooperative units are making a lot of trouble in order to compete for priority. Among them, V. Telegdi sent the experimental results to the Physical Review. The editor believed that their experimental results were rushed and confused and suggested that they be revised and published in the next issue. However, he still withdrew from the American Physical Society for this reason.And Lederman[2,15,18], after learning about C. S. Wu's experiment, only "three days" time, "verified" the "parity non-conservation", and the degree of non-conservation in the data was as high as $100 \%[18]^{206-207}$.

There was also unhappiness between C. S. Wu and his cooperator with the US Bureau of Standards and Metrology. Before the results of the experiment came out, Wu had already written the experiment report. On the Sunday after the experiment was completed, four scientists from the National Bureau of Standards and Wu sat down and were preparing to report on the paper, only to see that $\mathrm{Wu}$ had produced a written paper. They were a little surprised that Wu wrote the report without having any discussion with them[5] ${ }^{188-191}$ [21].

C. S. Wu is the world's "authority" in the field of $\beta$-decay experiments, and he has done similar judgment experiments more than once. $\mathrm{Wu}$ also used her experimental results to make a "summary" of the research work on the $\beta$-decay theory in physics from 1934 to 1948, which "clarified" the previous "fallacy" and "denied" Konopinski-Uhlenbeck's theory "supports" and "verifies" Fermi's theory[36].

Under the special background at the time, the nationalities of T. D. Lee and C. N. Yang, as a result, both sides of the Strait of China rushed to publicize their scientific "achievements."On February 15, 1957, the former Soviet Union "Pravda" also published a long article, propagating the compound inversion theory proposed by Landau in a high-profile manner, 
making it clear that Landau was the first person to discover parity non-conservation [31].

Whether driven by setbacks or not, although Lee and Yang's dissertation "can win" the Nobel Prize, it is not a qualified doctoral dissertation.They put forward their hypothesis in their paper, which has now become a "classic". The abstract of the paper is a "masterpiece" of "cautious" statement. It is difficult to see from this that it is this paper that overturns a long-standing and extremely affirmative principle in physics[21 ${ }^{163}$.Really can't tell, is this a helpless affirmation or a disguised negative? The relationship between C. N. Yang, T. D. Lee and C. S. Wu is reminiscent of the attitude of Watson and Crick, who discovered the double helix structure of DNA, towards R. E. Franklin[42-44].

Based on the above facts, after research and analysis, we believe that, the assumption that parity is non-conserved in weak interaction, the process of being "verified" was too hasty and sloppy. Almost all relevant personnel and units were coerced to run all the way, without allowing enough time for the parties involved, industry insiders, bystanders, or award jury, so that everyone could calm down and calmly think about and examine the work of Yang, Lee and $\mathrm{Wu}$.

\section{When will the "broken parity" be "reunited"?}

The amazing experimental results shocked the international physics community. People began to think about why at this important historical turning point, it was precisely three physicists who "guided" the physics community, "solved" a "fundamental structure of physics theory" and made people's fundamental understanding a great " liberation"?The mystery of " $\theta-\tau "$ ", as C. N. Yang said, "At that time, particle physicists discovered that they were in a situation like a person groping for a way out in a dark room. He knew that in a certain direction There is a door that can get him out of trouble. But in which direction does this door open?" [45] When C. S. Wu's preliminary experiment results came out, C. N. Yang notified Oppenheimer by telegram, and Oppenheimer replied with only a few words: "Out of the room" $[2,18]$.

At this point, Pandora's Box was opened. The experiment of non-conservation of parity has caused nuclear physics experimenters from all over the world to rush to their laboratories to "repeat" this experiment or make related experiments triggered by this experiment, and various new results continue to emerge: For example, the parity of positron $\mathrm{Co}^{58}$ is not conserved $\mathrm{Co}^{58}+\mathrm{e}+\mathrm{v}, \mathrm{CP}$ conservation, $\mathrm{CP}$ failure, two-thousandths of CP failure, CPT joint conservation, two-component neutrino theory and other results, new concepts in physics are constantly updated. In the following years, as we should have seen, under the "guidance" of Yang, Lee, and Wu, other symmetry was also overturned. The collapse of the parity building caused an "avalanche," the domino effect, and The Goebbels effect has appeared, and there have been more than 100 similar experiments that can "prove" "parity non-conservation" in a 
short period of time[2,18,21].

The tragedy of science is that a beautiful hypothesis is overturned by an ugly fact. This is a story about a major discovery, and also a story in which the fine theories often encountered on the way forward are stifled by ugly facts. It is really difficult to express what kind of impact the amazing experimental results have on the physics community. We challenged, in fact destroyed, a belief that people cherish: mirror symmetry in nature. The experiment also shocked many theoretical physicists, including Pauli, who left a famous thesis: "I can't believe that God is a weak left-handed"[15]. When Felix Bloch talked about the problem of parity non-conservation in weak interaction, he said that if the experiment proved this statement, he would be willing to eat his hat $[2,15,18,21]$.

"Conservation of parity, why do physicists doubt it so reluctantly? However, with complex mathematical reasoning, it can be proved that if $\theta$ and $\tau$ are the same particle, this means that mirror symmetry should be discarded when describing their decay.In other words, we must abandon the law of conservation of parity"[34,35], Yang made this explanation in his award-winning speech. In fact, quantum physicists are happy to see that $\theta$ and $\tau$ are the same particle. The experimental results of non-conservation of parity in weak interactions are exactly what the neutrino two-component theory expects[21,46]. C. S. Wu later reviewed their experimental results and described it as "three birds killed in one stone". The three birds she was referring to were "parity conservation, charge conjugate invariance, and two-component neutrino theory"[21,47]. The neutrino mass of the neutrino two-component theory must be 0 , but the discovery of the phenomenon of neutrino oscillation proves that the mass of neutrinos is not 0 .

Only after this, people slowly realized that the weak interaction force formed an independent field, perhaps on the same level as gravitation, electromagnetic force, strong force and subnuclear force[2][18 $]^{196}$. Perhaps this is the real purpose. Lederman and Demokritos have a "magical" dialogue that travels through time and space, which is intriguing $[15]^{34-62}$

Demokritos: Don't misunderstand me. They are all "smart people." The result of their argument is nothing more than one or the other playing the banner of God. ...But who believes it? A small group of you? Some betrayers? Or do you all believe it?

Lederman: We all believe, at least all "smart" particle physicists believe, but this theory (quantum theory) has been accepted by all scientists to a considerable extent, and they believe us in this respect.

How familiar is the "Dream" dialogue between Demokritos and Lederman? Fools can't see the emperor's new clothes. D. H. Lawrencesaid, "I like relativity and quantum theory because I don't know anything about them"[15]. So, do those who strive to maintain, support, and actively popularize "relativity and quantum theory" really understand it? Quantum theory is 
not a religion, and if it is, funding is not so difficult to raise. To borrow an old saying: Never use a map as a territory[15 $]^{200-201}$. We don't know whether Einstein's theory of relativity is correct or not, but his understanding of the degree of human stupidity is a well-known saying: "There are only two things that are infinite, that is, the universe and human stupidity, and what I am not sure about is whether the universe is infinite."

"If a physical theory can only be expressed by a very complicated mathematical scheme, then there are reasons not to trust it", Dirac said[48]. Now the development of life sciences, there has been a "quantization" trend. Regarding the explanation of cell bioelectricity phenomenon, the ion theory proposed by Hodgkin and Huxley and the GHK equation and H-H equation[10,49-51] are used. The equation introduces too many and too complicated calculation formulas to make the problem change. It's very complicated. Interestingly, Hodgkin and Huxley have a teacher-student relationship, while Huxley is a mathematics student[52].

There cannot be two leaves that are exactly the same in the world, and it is also impossible for people to wading across the same river at the same time. This is a philosophical saying. In theory, the mirror experiment is impossible to achieve. The work of C. N. Yang, T. D. Lee and C. $\mathrm{S}$. Wu did not prove the parity conservation in weak interactions, nor the non-conservation of parity in weak interactions." God turned out to be a left-handed man", On January 21, 1957, after poor Pauli calmed down, he wrote an obituary for the parity conservation in a humorous style:"We have a sad duty to declare our many years dear female friend-Parity—after a brief painful experience of experimental surgery, she passed away peacefully on January 19, 1957." Bloch is lucky because he doesn't have a hat." In a sense, a fairly complete theoretical structure has been fundamentally shattered. We don't know how these fragments will come together again in the future," I. I. Rabi lamented[53][21] ${ }^{173-176 .}$

\section{Conclusion and Discussion}

We believe that $\theta$ and $\tau$ are two different charged particles, derived from the result of a precursor particle "divided into two". The " $\theta-\tau$ " mystery is a "man-made" mystery. The experimental program proposed by C. N. Yang et al. cannot be used as a positive evidence of whether the weak interaction parity is conserved, nor can it positively answer whether $\theta$ and $\tau$ in the " $\theta-\tau$ " mystery are the same particle; C. S. Wu's $\operatorname{Co}^{60} \beta$ decay experiment is a pseudo-mirror experiment. Whether the experimental result violates the conservation of parity is based only on the assumptions of C. N. Yang and T. D. Lee. In fact, experiments such as polarized $\mathrm{Co}^{60}$ have not overturned the law of conservation of parity[54-57]. The work of $\mathrm{C}$. N. Yang et al. have brought quantum physicists from the "Little black house" to the "Big black house" or "smaller black house". The right and wise choice is to go back through "the door that came in". 
The history of scientific development of mankind is not long, and the inspiration for many of the work of modern scientists comes from the enlightenment of religion. For example, in 1947, the King of Denmark awarded Niels Bohr with the Treasure Elephant Medal, and the central pattern of the medal adopted the Chinese "Tai Chi Diagram" $[18]^{211}$; In 1983, in the trial issue of the "International Social Sciences" magazine of the United Nations Educational, Scientific, Cultural and Health Organization, the front page illustration selected the "FuxiNuwa Picture" unearthed in Xinjiang, China. Because the DNA double helix structure is very similar to it, it was also titled "Metaplasia of all things"; In 2019, we proposed an origami windmill model of potassium ion channels. Afterwards, we found that the principle of the model was surprisingly similar to the Buddhist swastika and Taoist Tai Chi Diagram [8].

American John Campell and Martin Gardner speculated that[21] ${ }^{133}$, Yang and Lee's ideas might have been inspired by China's "Tai Chi diagram", Yang and Lee were very happy Exploring the roots from the profound source of Eastern wisdom, "The Book of Changes", thinking that some prophecies sometimes change people's thinking in new directions. On November 26, 1959, Yang and Lee asked the Book of Changes: "Will there be a breakthrough in elementary particle physics in the next twenty years?" When C. N. Yang visited Shanxi in 1992, he also wrote an inscription "Science and religion are one family"[33]. However, Yang said in a speech in 2004: "The Book of Changes has influenced the way of thinking of Chinese culture, and this influence is one of the important reasons why modern science has not sprouted in China." [41] $]^{412-421}$

Someone once said that the prophet has been waiting for you here for a long time when scientists climbed to the top of the mountain with all their hardships. In 1957, when T. D. Lee gave a speech at the Nobel Prize award ceremony, he once said: "We may make great progress in researching knowledge, but we must remember that even at the root of the Buddha's finger, we It is still very far from absolute truth" $[2]^{125}$. Some of our modern people's scientific inspirations have been expressly or imperceptibly inspired by the prophets, but they are rarely grateful and lack due awe for the prophets. Caesar's return to Caesar, God's return to God, Newton's must be returned to Newton.

Finally, it should be noted that this paper was written during the "epidemic" period, and the people and things involved in the article are a bit "special". Regarding whether to write or whether to publish after writing, I sincerely thank my relatives, friends and colleagues for reminding me many times. For this reason, the author has also asked himself many times, what is the purpose of writing this paper? In the end, I still followed my heart instead of selfishness, and decided to write it out and publish it publicly. With the development of science today, it is time for some contents to reform from the bottom. If an incident of "deliberately misrepresent" occurs in the scientific community, it is a disaster for mankind. 


\section{References:}

[1] Lee, T. D., \& Yang, C. N. (1956). Question of Parity Conservation in Weak Interactions. Phys. Rev., 104: 254-258.

[2] Ji, Cheng. (2010). Nobel Prize in China: Biography of Tsung-Dao Lee. Beijing: International Cultural Publishing Company.

[3] Wu, C. S., Ambler, E., Hayward, R. W., et al. (1957). Experimental Test of Parity Conservation in Beta Decay. Phys. Rev., 105(4):1413-1415.

[4] Wu, C. S. (2008). Discovery of parity non conservation. Science, 60(1): 32-36.

[5] Jiang, C. J. (1997). Chien-shiung Wu: First lady of Physical Science. Shanghai: Fudan University Press.

[6] Sun, Z. D. (2016). The theory of brain cell activation. Harbin: Heilongjiang Science and Technology Press.

[7] Sun, Z. D. (2019). Revision and Supplement of the Theory of Dove-Like Particles. Journal of US-China Medical Science, 16(4): 193-195.

[8] Sun, Z. D. (2019). Potassium Channel Origami Windmill Model. Journal of US-China Medical Science, 16(4): 1-4.

[9] Sun, Z. D. (2019). Interpretation of Action Potential Generation Mechanism in Cells by Potassium Channel “Origami Windmill” Model. Journal of US-China Medical Science, 16(4): 1-7.

[10] Sun, Z. D. (2020). Enlightening Point of View Based on Potassium Channel "Origami Windmill” Model. Journal of US-China Medical Science, 17(4): 109-131.

[11] Sun, Z. D. (2020). Interpretation of Action Potential Generation Mechanism in Cardiomyocytes by Potassium Channel “Origami Windmill” Model. Journal of US-China Medical Science, 17(4): 132-139.

[12] Sun, Z. D. (2020). Conservation Law of Cell Bioelectricity Membrane Area and Ion Inequality Equation Based on Potassium Channel "Origami Windmill” Model. Journal of US-China Medical Science, 17(5): 109-131.

[13] Franklin, A. (1979). The Discovery and Nondiscovery of Parity Nonconservation. Stud. Hist. Phil. Sci., 10(3): 201-257.

[14] Budde, R., Chretien, M., Leitner, J., et al. (1956). Properties of Heavy Unstable Particles Produced by 1.3-BeV ${ }_{\pi}^{-}$Mesons. Phys. Rev., 103(6): 1827-1836.

[15] Lederman, L., \& Teresi, D. (2003). The God Particle: If the Universe Is the Answer, What Is the Question? Mi, X. J. Shanghai: Shanghai Scientific Technical \& education Publishers.

[16] Garwin, R. L., Lederman, L. M., Weinrich, M. (1957). Observations of the Failure of Conservation of Parity and Charge Conjugation in Meson Decays: the Magnetic Moment of the Free Muon. Phys. Rev., 105(4): 1415-1417.

[17] Friedman, J. I., \& Telegdi, V. L. (1957). Nuclear Emulsion Evidence for Parity Nonconservation in the Decay Chain. Phys. Rev., 105(5):1681-1682.

[18] Yang, J. Y. (2011). Biography of Chen-Ning Yang (enlarged edition). Beijing: SDX Joint Publishing Company. [19] Yang, C. N. (1963). A brief history of fundamental particle discovery. Shanghai: Shanghai Science and Technology Press.

[20] Yang, C. N. (1957). Present Knowledge About the New Particles. Reviews of Modern Physics, 29(2), 231-235.

[21] Ji, C., Liu, H. Z., Teng, L. (2004). Debate and solution to the discovery of parity non conservation. Lanzhou: Gansu science and Technology Press.

[22] Xu, K. Z., Chen, X. J., Chen, H. F. (2015). Modern physics (3rd Edition). Hefei: China University of science and Technology Press.

[23] Fabri, E. (1954). A Study of $\tau$-Meson Decay. Nuovo Cimento, 11: 479.

[24] Dalitz, R. (1953). CXII. On the Analysis of Meson Data and the Nature of the Meson. Phil. Mag., 44: 1068.

[25] Lee, T. D., \& Orear, J. (1955). Speculations of Heavy Mesons. Phys. Rev., 100(3): 932-933.

[26] Alvarez, L. (1956). Proc. Sixth Ann. Rochester Conf. V28-30.

[27] Purcell, E. M., \& Ramsey, N. F. (1950). On the Possibility of Electric Dipole Moments for Elementary Particles and Nuclei. Phys. Rev., 78(6):807.

[28] Ramsey, N. F. (1956). Molecular Beams. Lodon: Oxford University Press.

[29] Wick, G. C., Wightman, A. S., Wigner, E. P. (1952). The Intrinsic Parity of Elementary Particles. Phys. Rev., 88(1):101.

[30] Rochester, G. D., \& Butler, C. C. (1947). Evidence for the existence of new unstable elementary particles. Nature, 160(4077):855.

[31] Huang, Q. Q. (2018). Political competition behind Science: The international battle for parity non conservation 
theory in 1957. Journal of philosophy of science and technology, 35(6): 86-91.

[32] Weyl, H. (2002). Symmetry. Feng, C. T., \& Lu, J. Z. Shanghai: Shanghai Scientific Technical \& education Publishers.

[33] Jiang, C. J. (2011). Beauty of norms and symmetry_-Biography of Chen-Ning Yang. Guangzhou: Guangdong Economic Publishing Press.

[34] Lee, T. D. (1958). Weak Interactions and Nonconservation of Parity. Science, 127(3298): 569-573.

[35] Yang, C. N. (1958). Law of Parity Conservation and Other Symmetry Laws. Science, 127(3298): 565.

[36] Lee, T. D. (1997). Chien-shiung Wu and parity non Conservation Experiment. Science, 05(49): 4-12.

[37] Huang, Z. X. (2019). Amazing: wonderful mathematical theorems in mechanics - Noether theorem. Journal of mechanics and Practice, 41(5): 628-631.

[38] Yang, C. N. (1985). Forty years of learning and teaching. Hong Kong: Joint Publishing(H.K.).

[39] Noether, E. (1918). "Invariante Variationsprobleme." Nachrichten von der Gesellschaft der Wissenschaften zu Göttingen, Mathematisch-Physikalische Klasse, 1918: 235-257.

[40] Gao, C. (1989). The principle of "symmetry dominating interaction" and its methodological significance-On Chen-Ning Yang's thought of scientific methodology. Journal of science and technology and Dialectics, 4: 43-4.

[41] Yang, C. N. (2018). Dawn Anthology. Weng, F. Beijing: SDX Joint Publishing Company.

[42] Franklin, R. E., \& Gosling, R. G. (1953). Molecular Configuration in Sodium Thymonucleate. Nature, 171(4356): 740-741.

[43] Watson, J. D. (1968). The Double Helix.Tennessee: Kingsport Press.

[44] Maddox, B. (2003). Rosalind Franklin: The Dark Lady of DNA. New York: HarperCollins US.

[45] Yang, C. N., \& Yang, J. Y. (2002). Biography of Chen-Ning Yang: a master of science sees man and the world. Haikou: Hainan Publishing Press.

[46] Wu, C. S. (1972). Beta Decay and Parity Nonconservation. B. Maglich ed. Adventures in Experimental Physics, volume $\gamma$. Princeton: World Science Communications, 101.

[47] Novick, R. (1988). Thirty years since parity nonconservation. Birkhäuser Boston.

[48] Dirac, K. (2009). Dirac: A Scientific Biography. Xiao, M., et al. Changsha: Hunan Science and Technology Press.

[49] Wang, B. Y. (1982). Neuroelectrophysiology. Beijing: People's education Press.

[50] Goldman, D. E. (1943). Potential, impedance, and rectification in membranes. J Gen Phys., 27:37-60.

[51] Hodgkin, A. L., \& Huxley, A. F. (1952). Current carried by sodium and potassium ions through the membrane of the giant axon of Loligo. The Journal of Physiology, 116(4): 449-472.

[52] Gu, F. J. (2018). Hodgkin: Maxwell of Neuroscience. Scientific world, 12: 4.

[53] Bernstein, J. (1962). A Question of Parity. New Yorker, 38:49-104.

[54] Ge, X. C. (1991). Solution of Dirac equation and second excitation of vacuum. Journal of Changsha Normal University of Water Resources and Electric Power, 6(1): 75-83.

[55] Ge, X. C. (1994). Dirac equation and two component neutrino theory. Natural Science Journal of Changsha Normal University of Water Resources and Electric Power, 009(002):128-135.

[56] Ge, X. C. (1989). Neutrinos did not break the law of conservation. Journal of Huaibei Teachers College (Supported by Coal Industry) (Natural Science Edition), 10(03):17-25.

[57] Ge, X. C. (1984). On parity destruction and CP conservation. Journal of Huaibei Teachers College (Supported by Coal Industry), 1, 2: 17-24. 